\title{
Factors, trends and long-term outcomes for stroke patients returning to work: the South London Stroke Register (SLSR)
}

*Arup Sen, MRCP2, *Alessandra Bisquera, MSc1 ${ }^{1}$, Christopher McKevitt, PhD1,2, Anthony Rudd, FRCP ${ }^{1,2}$ Charles D.A. Wolfe, MD, FFPHM ${ }^{1,2}$, Ajay Bhalla, MD ${ }^{1,2}$

1. School of Population Sciences and Health Services Research, King's College London, London, UK

2.NIHR Biomedical Research Centre, Guy's and St Thomas' NHS Foundation Trust and King's College London, London UK

*Authors contributed equally

\section{Background}

Loss of productivity contributes to the economic burden of stroke. There is limited information on factors, trends and outcomes in return to work at different time-points post-stroke. This study aims to identify these in a multiethnic urban population.

\section{Methods}

Patterns of return to work were identified in individuals in paid work prior to first-ever stroke in the population based SLSR between 1995-2014. Employment status and outcomes including Barthel Index (BI) were assessed at 1, 5 and 10 years post-stroke. Multivariable logistic regression examined associations between patient characteristics and return to work.

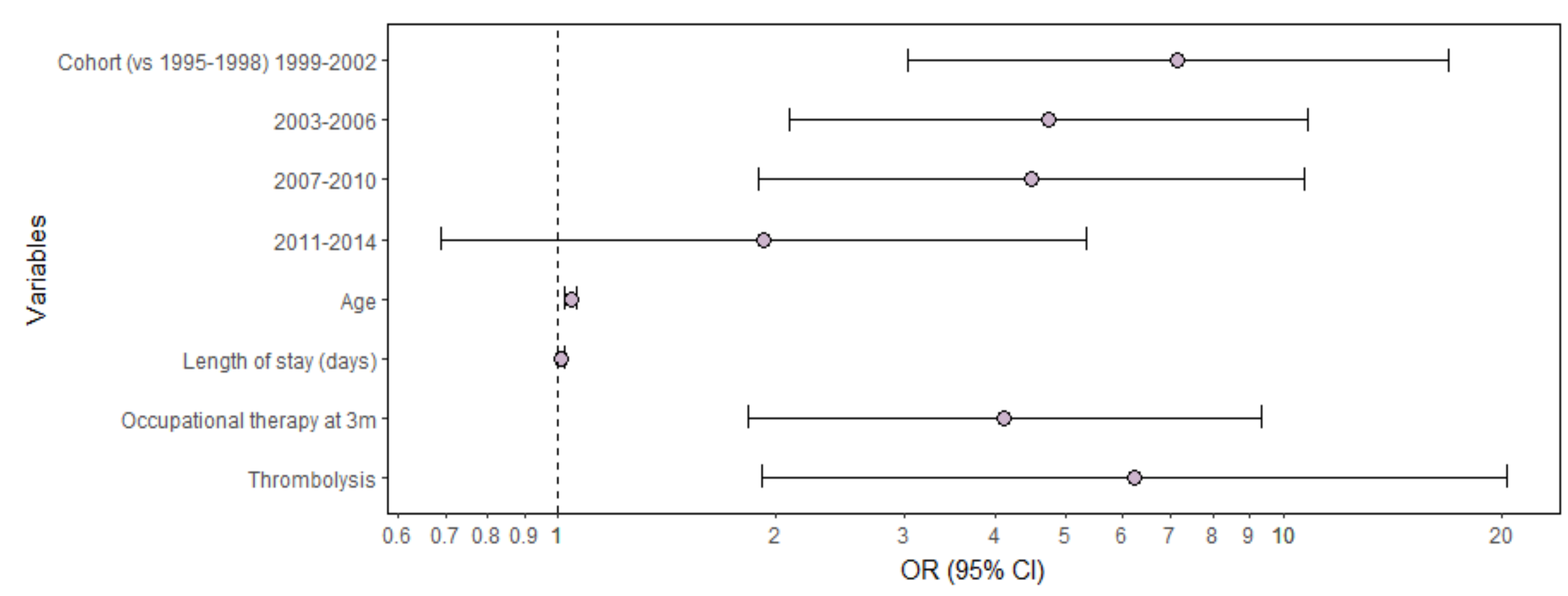

Fig 2. Odds of returning to work at 1 year post-stroke (adjusted for year cohort, age, smoking status, Barthel Index 7 days post-stroke, stroke severity (Glasgow Coma Scale, motor deficit), stroke unit treatment, length of stay, physiotherapy and occupational therapy 3 month post-stroke, and thrombolysis).

\section{Conclusions}

- A large proportion of stroke survivors remain unemployed despite functional independence, however this proportion reduces with time

- Return to work after stroke is associated with negative emotional consequence in the long-term

- Important predictive factors related to vocational outcome have been identified with regards to acute (intravenous thrombolysis) and post-acute (occupational therapy and physiotherapy) stroke care, which are both areas where previous research has been scarce

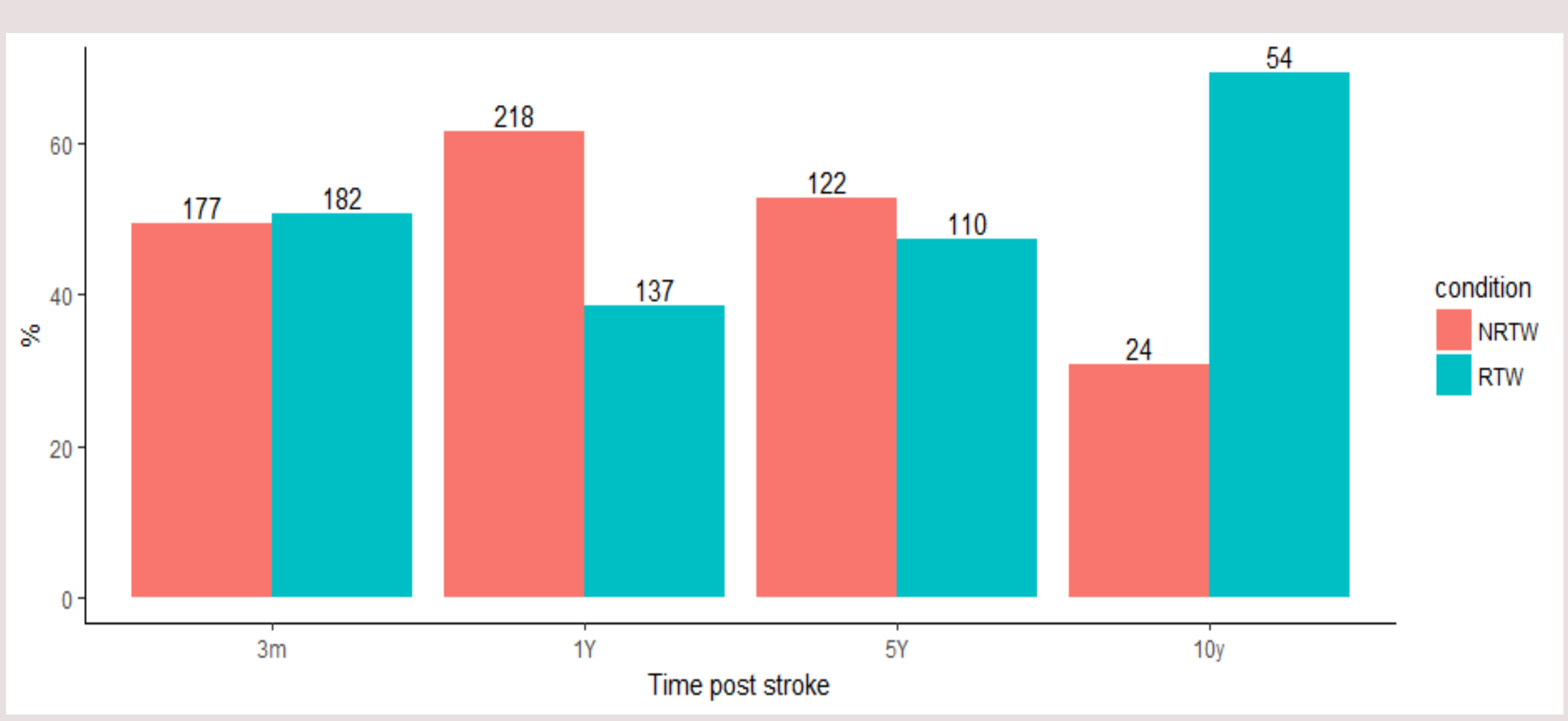

Fig 1 . Functionally independent $(\mathrm{BI}>19)$ patients who returned to work at $3 \mathrm{~m}, 1,5$ and 10 years post-stroke $($ RTW $=$ return to work, NRTW $=$ no return to work)

\section{Results}

- Among 940 patients working prior to their stroke, 34\% of patients were in employment at 3 months poststroke, declining to $27 \%$ at one year, $25 \%$ at 5 years and $10 \%$ at 10 years

- Factors associated with return to work at one year included receiving thrombolysis $(\mathrm{P}<0.01)$, occupational therapy $(\mathrm{P}<0.01)$, increasing age $(\mathrm{P}<0.001)$ and the cohorts of 1999-2002, 2003-2006 and 2007-2010 $(\mathrm{P}<0.001)$

- Patients returning to work at 1 year were more likely to return at 5 years (OR: 7.6; 95\% CI: 3.5-16.2) and 10 years (OR: 3.8; 95\% CI: 1.0-14.5)

- Of the patients who were independent at follow-up (BI>19), only $39 \%$ returned to work at 1 year, $47 \%$ at 5 years and $69 \%$ at 10 years

- There were higher rates of anxiety in patients working at 1 and 5 years post-stroke $(\mathrm{p}<0.01$ and $\mathrm{p}<0.05$ respectively)

- Similarly, there were higher rates of depression in patients working at 1 and 5 years post-stroke $(\mathrm{p}<0.001$ and $\mathrm{p}<0.01$ respectively)

Contact details: Arup Sen and Alessandra Bisquera Arup.Sen@gstt.nhs.uk, Alessandra.Bisquera@kcl.ac.uk Guy's and St Thomas' NHS Foundation Trust and King's College London, London SE1 1UL, UK

\section{School of Population Health \& Environmental Sciences}

